\title{
The Effect of High Ship Speed Ratio on Collision Avoidance Behavior of COLREGS
}

\author{
X.H. Wang, L. Li \& G. Chen \\ Jimei University, Xiamen, China
}

\begin{abstract}
The speed ratio is an important factor that must be considered when two vessels will course change to avoid collision. In the process of the research on Personifying Intelligent Decision-making for Vessel Collision Avoidance (short for PIDVCA), it is found that the effect of collision avoidance based on the existing "International Regulations for Prevention Collision at sea" (short for COLREGS) is greatly affected by the high speed ratio $(\mathrm{k}=\mathrm{Vt} / \mathrm{V} 0 \geqslant 1.5)$. Through the analysis on the geometric change law of two vessels' relative motion in Open waters, the effects of the responsibility for the ship collision avoidance under the COLREGS and special case for high-speed ratio is discussed. According to the collision avoidance measures taken for two vessels encounter situation, some reasonable suggestions are put forward and the simulation experiments that based on ship's intelligent collision avoidance simulation platform are given to support the idea.
\end{abstract}

\section{INTRODUCTION}

COLREGS which regulates the action of the ships' collision avoidance in the encounter situation, action rules, the distribution of rights and obligations of avoidance and so on, is the guarantee of safe and orderly navigation, prevention and reduction of collision of ships, and the maritime traffic rules that ship drivers should abide by. In recent years, with the further application of automation technology in the field of navigation and with the gradually development of intelligent ships represented by unmanned boats, research on intelligent decisionmaking for vessel collision avoidance is also developing rapidly. However, whether the intelligent decision-making for vessel collision avoidance can be applied in the real ships and accepted by the sailors depends on whether the decision-making follows the COLREGS and has the ability to simulate the ordinary practices of outstanding crews and reflect their excellent seamanship. COLREGS is the essential criterion to decision-making, in the process of refining avoidance strategies under different encounter situations; it is found that the effect of avoidance according to the rules is greatly affected by the high ship speed ratio. Through the analysis on the geometric change law of two vessels' relative motion in Open waters, the effects of the responsibility for the ship collision avoidance under the COLREGS and special case for high-speed ratio is discussed. According to the collision avoidance measures taken for two vessels encounter situation, some reasonable suggestions are put forward and the simulation experiments that based on ship's intelligent collision avoidance simulation platform are given to support the idea. 


\section{PIDVCA INSTRUCTIONS}

\subsection{Goal and Principle of PIDVCA}

The aim of PIDVCA algorithm is to provide crews safe, scientific and economic decision-making for vessel collision avoidance by a machine (computer), which can follows the COLREGS and has the ability to simulate the ordinary practices of crews and reflect their seamanship. Its security is reflected in the output of the avoidance decision that is premised on the maximized DCPA, and its scientific is embodied in the organic integration of quantitative analysis of vessels, relative motion geometric graphic and qualitative analysis of COLREGS and expert empirical knowledge, and its economy is reflected in its minimized track offset, while satisfy the precondition of the safety.

In the co-existence stage of manned and unmanned ships, for the PIDVCA can be accepted by the sailors to achieve concerted avoidance between manned ship and unmanned ship, algorithm's principles are as follows.

\section{First, follow the spirit of COLREGS.}

Second, simulate the ordinary practices of crews and reflect their excellent seamanship.

Thirdly, reflect the concretization, clearness and reasonable extension about important concepts and provisions of the COLREGS in practice.

COLREGS is very clear about the rules of conduct of two vessels in any condition of visibility, and there is no specific advice or specific method for collision avoidance of multiple vessels, only the words" ordinary practice of seamen, excellent seamanship" are used. According to crew training and expert consultation, " ordinary practice of seamen" means the making-decisions for collision avoidance of multiple vessels that can be widely accepted by crews, for example, adopt relevant requirements of COLREGS about two vessels to avoid key vessel of the multiple vessels, encounter situation. "seamanship of crews" is short for excellent seamanship is expressed in excellent crews making reasonable and effective avoidance decisions, concrete embodiment in the correctly evaluation of risk of collision, safety distance approaching (SDA), avoidance time, avoidance measure and resume time, as well as in the flexible reaction capacity to handle complicated situation.

\subsection{PIDVCA algorithm composition}

To the problem that the collision situation is endless and unrepeatable in the navigation due to the vessels different types, scales, speeds, sailing waters and the angles of the two vessels' rendezvous, PIDVCA adopts the personalized machine learning method to realize the purpose of automatic target perception, cognitive target, generation, verification and optimization decision.

According to the principle, PIDVCA consists of the initial PIDVCA generation algorithm and the PIDVCA verification and optimization algorithm. The initial PIDVCA generation algorithm is composed of the target intercrossing characteristics recognition algorithm, target potential danger judgment algorithm, encounter attribute recognition algorithm, the ship's avoidance attribute recognition algorithm for dangerous targets, encounter situation analysis and classification algorithm, dynamic hazard assessment algorithm and PIDVCA plan generation algorithm. PIDVCA verification and optimization algorithm is made up of predicting target potential risk analysis algorithm, ordinary algorithm of simulation of ordinary practice and seamanship algorithm of excellent seamanship, space searching optimization algorithm, algorithm of space-time searching optimization, algorithm of coordination collision avoidance optimization, PIDVCA plan local dynamic optimization algorithm, PIDVCA generation algorithm and emergency decision algorithm of dangerous situation.

PIDVCA algorithm is divided into two-vessel and multi-vessel PIDVCA generation and optimization algorithm considering two-vessel and multi-vessel encounter situation. For the two-vessel PIDVCA generation and optimization algorithm, the initial PIDVCA generation algorithm can basically achieve satisfactory avoidance effect, When necessary, it is supplemented by coordinated collision avoidance decision optimization algorithm, spatial searching optimization algorithm and PIDVCA plan dynamic optimization algorithm under the guidance of dynamic optimization objective function. For example, in Condition of restricted Visibility, when the own-ship avoids the target ship near the left beam, it needs to be supplemented by the local PIDVCA plan dynamic optimization algorithm to avoid producing a large track offset. For the PIDVCA generation and optimization algorithm of multivessel, it is necessary to perform the prediction target ship potential risk analysis algorithm of PIDVCA verification and optimization algorithm, and supplemented by other algorithms when necessary.

\section{ANALYZE OF THE RELATIVE MOTION GEOMETRIC CHANGE RULES OF CROSSING IN CONDITION OF NVISIBILITY}

\subsection{Encounter situation description}

Taking the crossing situation and in the Open waters as an example, it is assumed that the two vessels have the same scale and different speeds, and the affecting of vessels' maneuvering are ignored, and the SDA between the two vessels is the same, set as 1 sea mile, and the DCPA is zero. In order to facilitate the comparative analysis, the two vessels are each other's target-vessels and alter course to right for a vessel.

FIG. 1 and 2 are geometric diagrams of relative motion of two vessels in the same crossing situation as their left crossing and right crossing. That is, when the portside vessel as the own-ship, the other vessel on her right, and when the starboard vessel is the own-ship, the other vessel on her left. For ease of comparison, two independent relative motion geometries are presented in the one geometry. In the figures, $\mathrm{C} 0$ and $\mathrm{V0}$ respectively represent the heading and velocity of the own-ship, $\mathrm{Vt}$ and $\mathrm{Vr}$ respectively represent the true and relative velocity of the targetship, and AC is the turning angle. 




Figure 1. Geometric diagram of relative motion of a faster give-way vessel and a lower stand-on vessel's alter course as own-ship respectively to alter course



Figure 2. Geometric diagram of relative motion of a lower give-way vessel and a faster stand-on vessel as own-ship respectively to alter course

\subsection{Analysis figures}

As shown in figure 1, if the own-ship is the one vessel that faster and Give-way, and alters course to starboard for another vessel.

1 If the own-ship alters course at an equal distance and the target-ship's angle remains unchanged, the greater the turning angle $\mathrm{AC}$ is and the larger DCPA value will be, and with the decrease of the ship speed ratio $\mathrm{k}=\mathrm{Vt} / \mathrm{V} 0$ value, the smaller the steering Angle AC is required, if you want to get the same DCPA value.

2 If alter course of the own-ship remains unchanged, the larger the target-ship's angle is, the greater the DCPA value will be. With the decrease of ship speed ratio $\mathrm{k}$ value, the smaller the DCPA value, and the less effective the avoidance is.

If the own-ship is the one vessel that is slower and should Stand-on, and alters course to starboard for another vessel.

1 If the own-ship alter course at an equal distance and the target-ship's angle remains unchanged, the greater the turning angle $\mathrm{AC}$ is and the larger DCPA value will be, and with the increase of the ship speed ratio $\mathrm{k}$ value, the bigger the steering Angle AC is required to obtain the same DCPA value.

2 If alter course of the own-ship remains unchanged, the larger the target-ship's angle is, the greater the DCPA value will be. With the increase of ship speed ratio $\mathrm{k}$ value, the smaller the DCPA value, and the less effective the avoidance is.

As shown in figure 2, the lower Stand-on Vessel as the own-ship and alter course to right for another vessel. The give-way vessel is a lower ship and the stand-on vessel is faster ship. If the faster Stand-on Vessel as the own-ship and alter course to starboard for another vessel.

1 If the own-ship alters course at an equal distance and the target-ship's angle remains unchanged, the greater the turning angle $\mathrm{AC}$ is and the larger DCPA value will be, and with the increase of the ship speed ratio $\mathrm{k}$ value, the smaller the steering Angle AC is required to obtain the same DCPA value.

2 If alter course of the own-ship remains unchanged, the larger the target-ship's angle is, the greater the DCPA value will be. With the decrease of ship speed ratio $\mathrm{k}$ value, the smaller the DCPA value, and the better effective the avoidance is.

If the lower Give-way Vessel as the own-ship and alter course to starboard for another vessel.

1 If the own-ship alter course at an equal distance and the target-ship's angle remains unchanged, the greater the turning angle $\mathrm{AC}$ is and the larger DCPA value will be, And the larger the ship speed ratio is, the larger the Angle needed to change;

2 If alter course of the own-ship remains unchanged, the greater the speed ratio is, and the worse effective the avoidance is.

\subsection{Analysis results}

Although the encounter of two vessels is just a trivial case, it can help us to anlysis whether the decisionmaking is reasonable and how to optimize.

In conclusion, COLREGS is completely reasonable for the two vessels in sight of one another to divide the Give-way and the Stand-on vessel according to regions except the situation of overtaking, but in the case of crossing abeam situation, only when the speed of Stand-on Vessel is smaller or the speed ratio of the two vessels is not much different $(\mathrm{k}=\mathrm{Vt} / \mathrm{V} 0)<1.5)$, the effective of the avoidance would seem satisfactory. When the speed ratio is $(\mathrm{k}=\mathrm{Vt} / \mathrm{V0})>=1.5$, if avoid target-ship according the COLREGS, there are unreasonable effective of the avoidance.

\section{SIMULATION EXPERIMENT AND DECISION- MAKING OPTIMIZATION}

In order to make the decision-making of collision avoidance more safe, scientific and economic, a lot of simulation experiments of PIDVCA algorithm were done on the intelligent control simulation platform that is approximate the real ships. It is found that there is unreasonable decision-making of avoidance collision that strictly perform the COLREGS in the condition of ship speed ratio $(\mathrm{k}=\mathrm{Vt} / \mathrm{V} 0)>=1.5$. As shown in figure 3 , in visibility, according the COLREGS, the encounter situation is the crossing, and target-ship 1 is the give-way own-ship, and the simulation results of avoidance collision is not good. The bigger ship speed ratio $\mathrm{k}$ is, and the worse the result becames. This is, the collision avoidance action of the lower ship will be far earlier than the faster ship, and the Leads depend on the value of $\mathrm{Vt} / \mathrm{V} 0$, the bigger $\mathrm{k}$ is and the Leads gets larger. 
A crossing left near the beam encounter situation in restricted visibility is taken as an example to illuminate the effect of high speed ratio on collision avoidance behavior of COLREGS. According to the COLREGS, the rules of conduct of vessels in restricted visibility are that every vessel shall proceed at a safe speed adapted to the prevailing circumstances and conditions of restricted visibility. A power-driven vessel shall have her engines ready for immediate maneuver. This is, in restricted visibility, every vessel have responsibility to undertake obligation of avoidance. It is unreasonable for the lower stand-on own-ship, from which the target-ship is coming left, especially for the crossing left near the beam $\left(247.5^{\circ}<\right.$ relative orientation of target-ship $<292.5^{\circ}$ ).



Figure 3. Simulation experiments trajectory of avoidance collision in crossing situation and in sight of one another $(\mathrm{Vt} / \mathrm{V} 0=1.8, \mathrm{SDA}=1.99$ )

From the above analysis results, in condition of visibility, the avoidance moment of lower stand-on vessel is far earlier than the higher give-way vessel. In condition of restricted visibility, in order to avoid dangerous, "the lower stand-on vessel in visibility" will choose to take avoidance action first, and when the avoidance time of "the higher give-way vessel in visibility" arrives, it will choose not to take collision avoidance action for disappearing of the risk of collision, so that and two vessels have long standoff, because of the smaller relative speed, As shown in figure 4 (a). Obviously, this kind of collision avoidance action does not conform to the safety, science and economy of navigation of ships.

In order to solve the above problems, and satisfy the premise of scientific, safe and the minimum track offset, it's integrated PIDVCA algorithm to dynamic optimize decision-making of collision avoidance, and the optimization results is shown in figure 4 (b) and $4(\mathrm{c})$. Optimization approach in figure 4(b) is to make "the lower stand-on vessel in visibility" perform avoidance action in condition of restricted visibility according to the last opportunity of steering rudder of "the lower stand-on vessel in visibility". In figure 4(c), Optimization approach is that "the higher give-way vessel" will take the initiative to fulfill their obligation to give way in advance in condition of restricted visibility, so that "the low-speed stand-on vessel in visibility" will remain stand on for disappearing of the collision dangerous.

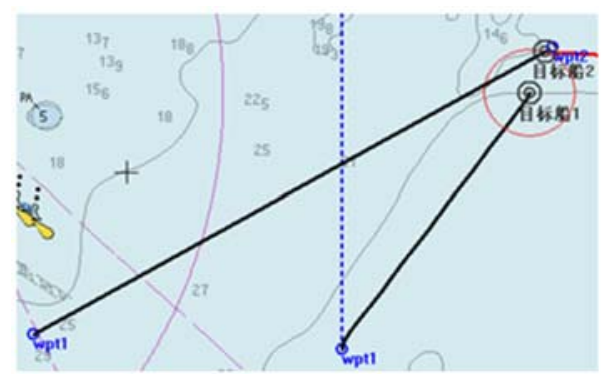

(a) Decision-making optimization before

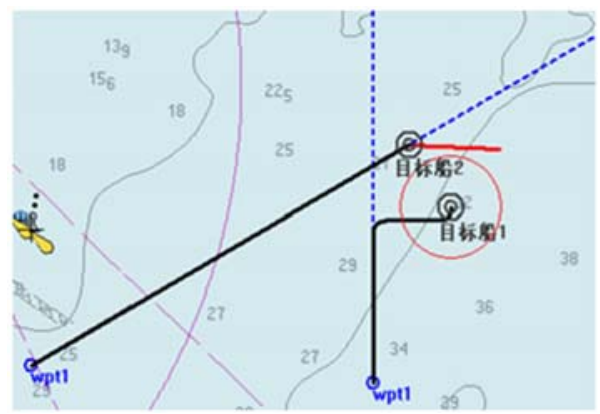

(b) Optimized decision-making of "the lower stand-on vessel in visibility"

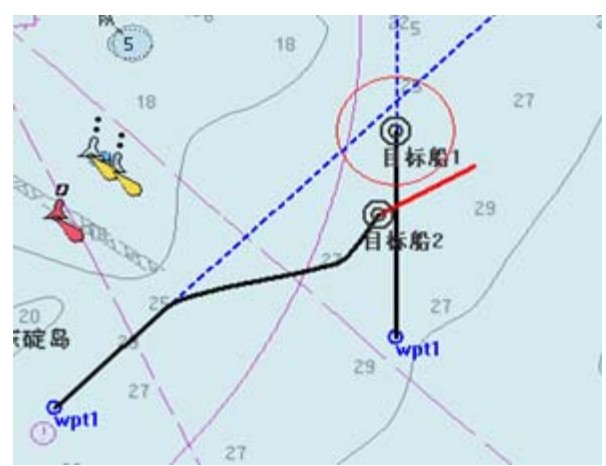

(c) Optimized decision-making of "the higher give-way vessel in visibility"

Figure 4. Simulation experiment results of before and after optimization of decision-making for the left near the beam encounter situation and in restricted visibility

The above simulation examples show that if "the lower stand-on vessel in visibility" take action to avoid collision strictly according to COLREGS in condition of restricted visibility, there is inharmonious situation. If "the higher give-way vessel" can take on more responsibility for avoidance collision, the avoidance dangerous will be better.

\section{CONCLUSIONS AND SUGGESTIONS}

Whether it is to provide auxiliary decision-making for manned ship or automatic decision-making of collision avoidance for intelligent ship or unmanned ship, intelligent decision-making for vessel collision avoidance must follow the spirit of COLREGS and can simulate the ordinary practices of crews and reflect their seamanship. However, with the rapid development of artificial intelligence and computer technology, vessels are developing in the direction large-scale, high-speed and intelligence, and it makes some rules of COLREGS unreasonable. For example, the action of avoidance collision only is based on the relative bearing of approaching vessels, there is no the ship speed ratio to be taken into account, which is reasonable in the years that there was little difference 
of speed between power-driven vessel. In the moderns, the high-speed vessel are ubiquitous, the ship speed ratio should be taken into account. The effects are summarized below.

1 In visibility and in right crossing situation, if the stand-on and give-on vessel has a high speed ratio, there is a less effective avoidance collision according to COLREGS. Namely, avoidance opportunities of the give-way vessel will become earlier which depend on the Vt/V0. The larger $\mathrm{k}$ is, and the earlier avoidance opportunities is.

2 In restricted visibility and in crossing near the left beam situation, the own-ship as the lower vessel act to avoid according to COLREGS, there is a less effective avoidance also, maybe more dangerous.

In view of these influences, it is suggested that the research of intelligent collision avoidance can reasonably extend the COLREGS to solve the unreasonable phenomenon of avoidance effect caused by strictly avoid collision according to COLREGS.

\section{ACKNOWLEDGMENT}

Project supported by the General Program for the National Natural Science Foundation of China (Grant no. 51879119).

\section{REFERENCES}

Wu Z.L. 1985. Course changes to avoid collision as a function of the speed ratio. Dalian: Journal of Dalian Marine College. 11(1):15-22.

Li L.N. et al. 2014. Review of the research on Personifying Intelligent Decision-making for Vessel Collision Avoidance. Shanghai: Marine Technology.2014 (2):42-49.

Chen G.G. 2016.Key technology of the research on ship avoidance collision automation. Dalian Marine College

Li L.N. et al. 2011. Construction of the PIDVCA system and its evaluation standard. Dalian: Journal of Dalian Marine College. 37(4):1-6

Li L.N. \& Zhang S.G. 2012. Maritime automation. China communications press 